“C 2015 IEEE. Personal use of this material is permitted. Permission from IEEE must be obtained for all other uses, in any current or future media, including reprinting/republishing this material for advertising or promotional purposes, creating new collective works, for resale or redistribution to servers or lists, or reuse of any copyrighted component of this work in other works." 


\title{
Energy Efficient Cooperative Transmission in Single-Relay UWB Based Body Area Networks
}

\author{
Jie Ding*, Eryk Dutkiewicz*, Xiaojing Huang ${ }^{\dagger}$, Gengfa Fang* \\ ${ }^{*}$ Department of Engineering, Macquarie University, Australia \\ \{jie.ding1, eryk.dutkiewicz, gengfa.fang\}@mq.edu.au \\ $\dagger$ University of Technology, Sydney \\ xiaojing.huang@uts.edu.au
}

\begin{abstract}
Energy efficiency is one of the most critical parameters in ultra-wideband (UWB) based wireless body area networks (WBANs). In this paper, the energy efficiency optimization problem is investigated for cooperative transmission with a single relay in UWB based WBANs. Two practical onbody transmission scenarios are taken into account, namely, along-torso scenario and around-torso scenario. With a proposed single-relay WBAN model, a joint optimal scheme for the energy efficiency optimization is developed, which not only derives the optimal power allocation but also seeks the corresponding optimal relay location for each scenario. Simulation results show that the utilization of a relay node is necessary for the energy efficient transmission in particular for the around-torso scenario and the relay location is an important parameter. With the joint optimal relay location and power allocation, the proposed scheme is able to achieve up to 30 times improvement compared to direct transmission in terms of the energy efficiency when the battery of the sensor node is very limited, which indicates that it is an effective way to prolong the network lifetime in WBANs.
\end{abstract}

\section{INTRODUCTION}

Wireless body area network (WBAN) is a promising technology that can improve healthcare quality with lightweight sensors on or in the human body [1]. Ultra-wideband (UWB) technology has great potential for applications in WBAN, owing to its simple electronics and low power consumption. In UWB based WBANs, power resource for sensor nodes is very limited due to stringent constrains in size and weight, and in most cases batteries are not rechargeable. Therefore, to maximize the lifetime of WBANs, one of the key issues in WBANs is energy efficiency. On the other hand, relay assisted cooperative communication has drawn much attention in wireless sensor networks (WSNs) which can improve the energy efficiency effectively [2]-[4].

Unlike WSNs, UWB based WBANs consist of some unique properties, e.g., analog transmission, distinct channel characteristics and limited network size. It is inadequate to apply existing techniques on the energy efficiency in WSNs to WBANs directly. Thus, the energy efficiency problem in UWB based WBANs is still an open issue. Some studies have been conducted in the relay assisted WBANs regarding the energy efficiency [5]-[8]. In [5] and [6], the optimal power allocation with the constraint of target outage probability was studied to minimize the average power consumption for onbody transmissions. Both works demonstrate that cooperative communication can improve the energy efficiency in WBANs. However, the impact of the relay location is not considered. In [7], relay nodes are utilized to minimize the power consumption while providing the necessary reliability for inbody transmissions. In [8], topology design in WBANs was investigated to increase the network lifetime. Both works have shown that the relay location is an influential parameter for system performance in WBANs. In fact, the signal strength is mostly affected by the physical location of the nodes in relation to each other as well as the human body in WBANs. Therefore, deploying relay node optimally in terms of the optimal location in WBANs is essential.

Based on this motivation, we extensively evaluate the energy efficiency in single-relay assisted UWB based WBANs by considering the joint optimal relay location (RL) and power allocation (PA). In this paper, two on-body cooperative transmission scenarios are investigated, namely, along-torso scenario and around-torso scenario. To achieve the maximum energy efficiency for each scenario, a relay-location based network model is proposed first, and then a joint optimal scheme is developed to seek the relay with the optimal location, together with the corresponding optimal power allocation. The implementation of the proposed scheme is highlighted afterward. Simulation results show that the relay location has a notable impact on the energy efficiency. With the joint optimal relay location and power allocation, the proposed scheme outperforms other single-relay transmission schemes. Moreover, up to 30 times improvement on the energy efficiency can be achieved compared to direct transmission in particular for the around-torso scenario when the battery of the sensor node is very limited, which indicates that the proposed scheme is able to prolong the network lifetime and extend the transmission range in WBANs significantly. In practice, the proposed scheme can provide an insight into the design of healthcare applications with respect to the proper placement of the relay node along with the optimal transmit power level in WBANs.

\section{System AND Channel Models}

\section{A. System Scenarios}

In this paper, we consider a WBAN which is composed of three types of nodes: one wearable sensor node, one 
body network coordinator, and one relay node. The sensor node is used to monitor the physiological states of a person periodically, e.g., measuring the body temperature, heartbeat or recording body activities, and it is connected to the coordinator directly or through the relay node. Normally, the coordinator is a personal digital assistant (PDA) attached on the human body. Note that the type and position of the sensor node in a WBAN depend on the requirement of patient.

In this work, a single-relay cooperative transmission is considered in UWB based WBANs, in which a relay node $R$ is employed to assist a sensor node $S$ (Source) to communicate with the coordinator $D$ (Destination). We herein study a typical two-phase amplify and forward (AF) cooperative protocol. It consists of two time slots with equal duration, in which $S$ broadcasts its signal to $D$ and $R$ during the first time slot, and in the second time slot, $R$ forwards its received signal to $D$. We assume that $S$ and $R$ are both on-body nodes and they are always located on the same side of the human body. For the on-body cooperative transmission, two practical scenarios are investigated, namely, along-torso scenario and around-torso scenario. In the along-torso scenario, the condition that $S$ and $D$ are on the same side of the human body is applied, while the condition that $S$ and $D$ are on the different sides of the human body is applied in the around-torso scenario.

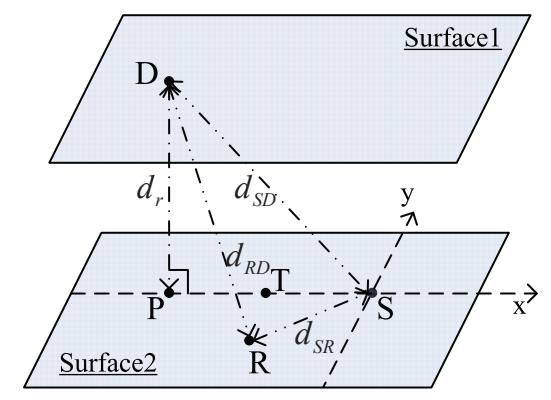

Fig. 1. Proposed cooperative model for WBANs.

In Fig. 1, a relay-location based cooperative model is proposed. As shown in this figure, the around-torso scenario is considered. Without loss of generality, we assume that $D$ is located on the front side of the human body (Surface1) and $S$ and $R$ are located on the back side of the human body (Surface2). The distance between these two surfaces is $d_{r}$ $\left(d_{r} \geq 0\right) . d_{S D}, d_{S R}$, and $d_{R D}$ denote the distances from $S$ to $D, S$ to $R$ and $R$ to $D$, respectively. We denote the projection of $D$ on Surface 2 by point $P$ and point $T$ is located in the middle between $P$ and $S$. To represent the location of $R$, we construct a xy-plane on Surface2, where $S$ is set to be the origin point and the $\mathrm{x}$-axis is along $P$ to $S$. With a given coordinates $\left\{x_{r}, y_{r}\right\}$ for $R$, we have

$$
d_{S R}\left(x_{r}, y_{r}\right)=\sqrt{x_{r}^{2}+y_{r}^{2}}
$$

and

$$
d_{R D}\left(x_{r}, y_{r}\right)=\sqrt{\left(x_{r}+d_{S D} \sin \theta\right)^{2}+y_{r}^{2}+d_{r}^{2}}
$$

where $\theta=\arccos \left(d_{r} / d_{S D}\right)$.

It is noted that the equivalent cooperative model will be simplified to the one for the along-torso scenario when $d_{r}=0$. In this case, $D$ is located on the same surface with $S$. Thus, we can consider the along-torso scenario as a special case in the around-torso scenario. In the sequel, we focus on the energy efficiency optimization problem in the around-torso scenario and only detail the solution of the considered problem for the around-torso scenario.

\section{B. Channel Models}

Accordingly, two types of channel models are considered in UWB based WBANs for on-body cooperative transmission, namely, along-torso channel model and around-torso channel model. Since signals are transmitted over the along-torso channel for the $S-R$ link and over the around-torso channels for the other two links, both channel models are used in the around-torso scenario. Table I summarizes corresponding parameters for path loss models [9].

TABLE I

UWB BaSEd WBAN PaTH LosS MOdels For ON-BOdY

\begin{tabular}{|c|c|}
\hline along-torso channel & $P L_{0}^{d B}(d)=L_{0}+10 n_{0} \log _{10}\left(\frac{d}{d_{0}}\right)$ \\
\hline$L_{0}[\mathrm{~dB}]$ & 44.6 \\
\hline$n_{0}$ & 3.1 \\
\hline$d_{0}[m]$ & 0.1 \\
\hline \hline around-torso model & $P L_{1}^{d B}(d)=L_{1}+10 n_{1} \log _{10}\left(\frac{d}{d_{1}}\right)$ \\
\hline$L_{1}[\mathrm{~dB}]$ & 48.4 \\
\hline$n_{1}$ & 5.9 \\
\hline$d_{1}[m]$ & 0.1 \\
\hline
\end{tabular}

From the path loss models defined in the log scale, the path losses in the linear scale from $S$ to $D, S$ to $R$, and $R$ to $D$ in the around-torso scenario can be obtained as,

$$
P L_{S D}\left(d_{S D}\right)=10^{P L_{1}^{d B}\left(d_{S D}\right) / 10}=M_{1} d_{S D}^{n_{1}},
$$

$$
P L_{S R}\left(d_{S R}\right)=10^{P L_{0}^{d B}\left(d_{S R}\right) / 10}=M_{0} d_{S R}^{n_{0}}
$$

and

$$
P L_{R D}\left(d_{R D}\right)=10^{P L_{1}^{d B}\left(d_{R D}\right) / 10}=M_{1} d_{R D}^{n_{1}},
$$

respectively. $M_{0}=\left(1 / d_{0}\right)^{n_{0}} 10^{L_{0} / 10}$ and $M_{1}=$ $\left(1 / d_{1}\right)^{n_{1}} 10^{L_{1} / 10}$ are constant.

For all the links considered, the energy-normalized channel impulse response (CIR) can be written as

$$
h_{k}(t)=\sum_{l=0}^{L_{k}-1} \alpha_{l, k} \delta\left(t-\tau_{l, k}\right),
$$

where $k \in\{S D, S R, R D\}$ denotes the links from $S$ to $D, S$ to $R$ and $R$ to $D$ respectively. $L_{k}$ is the number of multipaths, $\tau_{l, k}$ is the delay of the $l$ th path, and $\alpha_{l, k}$ is the gain of the $l$ th path. Since real signals are employed in UWB systems, each path gain is real also. Further detail on the delay profile for along-torso and around-torso links can be found in [9]. 


\section{Energy Efficiency of Direct Transmission}

Without loss of generality, we present the IR-UWB signal transmission with pulse-amplitude modulation (PAM). When data is modulated, pulse shaped and transmitted repeatedly over $N_{f}$ consecutive frames at $S$, the received signal at $D$ is given by

$$
\begin{aligned}
r_{S D}(t)= & b \sqrt{\frac{P_{s}}{P L_{S D}\left(d_{S D}\right)}} \sum_{j=0}^{N_{f}-1} g_{S D}\left(t-j T_{f}\right) \\
& +n_{S D}(t), \quad b= \pm 1
\end{aligned}
$$

where $b$ is the transmitted symbol and $P_{s}$ is the transmit power of $S$ and the value of $P_{s}$ depends on the battery power limit of $S$. In this paper, we assume that $P_{s} \leq P_{\max }$, where $P_{\max }$ is the maximum transmit power for each transmit node, which is constrained by Federal Communications Commission (FCC) power spectral density (PSD) emission limit for UWB signals.

$$
g_{S D}(t)=\omega(t) * h_{S D}(t)=\sum_{l=0}^{L_{S D}-1} \alpha_{l, S D} \omega\left(t-\tau_{l, S D}\right),
$$

where $*$ represents convolution. $\omega(t)$ denotes the ultrashort pulse waveform with $T_{w}$ duration, which has the unit energy $\int_{t=0}^{T_{f}} \omega^{2}(t) d t=1 . T_{f}$ is the duration of frame and it is set to be large enough to avoid the inter-symbol interference (ISI). $n_{S D}(t)$ is the additive white Gaussian noise (AWGN) with zero mean and variance $\sigma_{n}^{2}$.

At $D$, a received pulse waveform matched filter is employed. After summing up all the outputs over $N_{f}$ frames, the decision statistic $b$ at $D$ can be written as

$$
b_{S D}=b N_{f} \sqrt{\frac{P_{s}}{P L_{S D}\left(d_{S D}\right)}} \bar{\xi}_{S D}+\hat{n}_{S D},
$$

where $\bar{\xi}_{S D}$ is the captured multipath energy during $T_{f}$ at $D$ in direct trasmission and $\hat{n}_{S D}$ is a white Gaussan noise with zero mean and variance $N_{f} \bar{\xi}_{S D} \sigma_{n}^{2}$.

With (5), the spectral efficiency (SE) for direct transmisison can be given by

$$
C_{S D}=\frac{1}{N_{f}} \log _{2}\left(1+\gamma_{S D}\right)
$$

where $\gamma_{S D}=\frac{N_{f} P_{s} \bar{\xi}_{S D}}{P L_{S D}\left(d_{S D}\right) \sigma_{n}^{2}}$ is the received signal to noise ratio $(\mathrm{SNR})$.

In this paper, we define the energy efficiency as the ratio of the SE over the total power dissipation (unit:bits/Joule/Hz). Thus, the energy efficiency of direct transmission can be written as

$$
U_{S D}=\frac{C_{S D}}{P_{S D}}=\frac{\log _{2}\left(1+\gamma_{S D}\right)}{N_{f}\left(\varepsilon P_{s}+P_{c t}+P_{c r}\right)},
$$

where

$$
P_{S D}=\varepsilon P_{s}+P_{c t}+P_{c r}
$$

is the power dissipation per frame. $\varepsilon$ is a constant which accounts for the inefficiency of the power amplifier. $P_{c t}$ is the power dissipation for transmitter circuit per frame and $P_{c r}$ is the power dissipation for receiver circuit per frame.

\section{ENERGY EFFICIENCY OF COOPERATIVE TRANSMISSION}

\section{A. Problem Formulation}

Similar to $D, R$ is also equipped with a matched filter. With the considered AF cooperative protocol, the SE of the singlerelay cooperative transmission can be given by

$$
C_{S R D}=\frac{1}{2 N_{f}} \log _{2}\left(1+\gamma_{1}+\gamma_{2}\right),
$$

where $\gamma_{1}$ is the received SNR at $D$ in the first time slot and

$$
\gamma_{2}=\frac{\gamma_{S R} \gamma_{R D}}{\gamma_{S R}+\gamma_{R D}+1} \approx \frac{\gamma_{S R} \gamma_{R D}}{\gamma_{S R}+\gamma_{R D}}
$$

is the received SNR at $D$ in the second time slot. $\gamma_{S R}$ and $\gamma_{R D}$ are the received SNRs for the links $S-R$ and $R-D$, respectively.

In the around-torso scenario, the expressions of $\gamma_{1}, \gamma_{S R}$ and $\gamma_{R D}$ are given by

$$
\begin{gathered}
\gamma_{1}=\frac{N_{f} P_{1} \xi_{S D}}{P L_{S D}\left(d_{S D}\right) \sigma_{n}^{2}}, \\
\gamma_{S R}=\frac{N_{f} P_{1} \xi_{S R}}{P L_{S R}\left(d_{S R}\right) \sigma_{n}^{2}}, \\
\gamma_{R D}=\frac{N_{f} P_{2} \xi_{R D}}{P L_{R D}\left(d_{R D}\right) \sigma_{n}^{2}},
\end{gathered}
$$

where $P_{1}$ and $P_{2}$ are the transmit power at $S$ and $R$, respectively. In this paper, to make a fair comparison with direct transmission, we assume that the total transmit power resource in cooperative transmission is no more than that in direct transmission, i.e., $P_{1}+P_{2} \leq P_{s} . \xi_{S D}, \xi_{S R}$, and $\xi_{R D}$ are the captured multipath energy during $T_{f}$ for the links $S-D$, $S-R$, and $R-D$ in cooperative transmission, respectively.

On the other hand, the average power dissipation per frame in cooperative transmission can be written as

$$
P_{S R D}=\frac{1}{2}\left(\varepsilon\left(P_{1}+P_{2}\right)+2 P_{c t}+3 P_{c r}\right) .
$$

Using (9) and (14), the corresponding energy efficiency for cooperative transmission can be expressed as

$$
U_{S R D}=\frac{C_{S R D}}{P_{S R D}}=\frac{\log _{2}\left(1+\gamma_{1}+\gamma_{2}\right)}{N_{f}\left(\varepsilon\left(P_{1}+P_{2}\right)+2 P_{c t}+3 P_{c r}\right)},
$$

Substituting (1), (2), and (11)-(13) into (9), we have

$$
U_{S R D}\left(x_{r}, y_{r}, P_{1}, P_{2}\right)=\frac{C_{S R D}\left(x_{r}, y_{r}, P_{1}, P_{2}\right)}{P_{S R D}\left(P_{1}, P_{2}\right)},
$$

where

$$
\begin{gathered}
C_{S R D}\left(x_{r}, y_{r}, P_{1}, P_{2}\right)=\frac{1}{2 N_{f}} \log _{2}(1+\underbrace{\frac{N_{f} P_{1} \xi_{S D}}{P L_{S D}\left(d_{S D}\right) \sigma_{n}^{2}}}_{\gamma_{1}\left(P_{1}\right)} \\
+\underbrace{\left.\frac{1}{\frac{P L_{S R}\left(d_{S R}\left(x_{r}, y_{r}\right)\right) \sigma_{n}^{2}}{N_{f} P_{1} \xi_{S R}}+\frac{P L_{R D}\left(d_{R D}\left(x_{r}, y_{r}\right)\right) \sigma_{n}^{2}}{N_{f} P_{2} \xi_{R D}}}\right)}_{\gamma_{2}\left(x_{r}, y_{r}, P_{1}, P_{2}\right)}
\end{gathered}
$$


Obviously, $U_{S R D}$ is the function of variables $\left\{x_{r}, y_{r}, P_{1}, P_{2}\right\}$. To obtain the maximum $U_{S R D}$ for cooperative transmission, we must find the optimal set $\left\{x_{r}^{o}, y_{r}^{o}, P_{1}^{o}, P_{2}^{o}\right\}$ that makes $U_{S R D}\left(x_{r}, y_{r}, P_{1}, P_{2}\right)$ achieve its maximum. Thus, the joint optimal RL and PA problem for the energy efficiency optimization in the around-torso scenario can be mathematically formulated as

$\underset{x_{r}, y_{r}, P_{1}, P_{2}}{\operatorname{maximize}} U_{S R D}\left(x_{r}, y_{r}, P_{1}, P_{2}\right)$

subject to $\mathrm{C} 1:\left(x_{r}+\frac{d_{S D} \sin \theta}{2}\right)^{2}+y_{r}^{2} \leq\left(\frac{d_{S D} \sin \theta}{2}\right)^{2}$,

$\mathrm{C} 2: x_{r} \leq \delta$,

C3 : $P_{1}+P_{2} \leq P_{s}$,

where $\mathrm{C} 1$ is imposed to guarantee that $R$ is only located in the circle centered at $T$ with radius $\frac{d_{S D} \sin \theta}{2}$. This special circle for $R$ is considered based on the fact that we can always find a corresponding relay location within the circle which can provide a better performance than those beyond the circle. $\mathrm{C} 2$ is imposed to guarantee that $\left\{x_{r}=0, y_{r}=0\right\}$ has to be beyond $\mathrm{C} 1$ since $R$ cannot coincide with $S$, where $|\delta|$ is a very small constant and we set $-10^{-6}<\delta<0$. With $\mathrm{C} 1$ and C2, we have $d_{S R}, d_{R D}<d_{S D}$.

\section{B. Joint Optimal RL and PA}

The problem in (18) is a nonlinear fractional programming problem. Based on the Theorem in [10], for the considered nonlinear fractional programming problem in (18), there exists an equivalent nonlinear parametric programming problem with an objective function in subtractive form, e.g. $C_{S R D}\left(x_{r}, y_{r}, P_{1}, P_{2}\right)-q^{o} P_{S R D}\left(P_{1}, P_{2}\right)$, and $q^{o}$ can be achieved if and only if $C_{S R D}\left(x_{r}^{o}, y_{r}^{o}, P_{1}^{o}, P_{2}^{o}\right)-$ $q^{o} P_{S R D}\left(P_{1}^{o}, P_{2}^{o}\right)=0$, where $q^{o}$ and $\left\{x_{r}^{o}, y_{r}^{o}, P_{1}^{o}, P_{2}^{o}\right\}$ are the maximum energy efficiency and the corresponding optimal RL and PA set, respectively. Thus, with the transformed problem, an iterative algorithm known as the Dinkelbach method [10] can be proposed for solving the energy efficiency optimization problem in (18) equivalently, which guarantees the convergence to the optimal energy efficiency. Table II summarizes the proposed iterative algorithm and for each iteration, the problem in (19) with a given $q$ need to be solved.

$$
\begin{array}{ll}
\underset{x_{r}, y_{r}, P_{1}, P_{2}}{\operatorname{maximize}} & C_{S R D}\left(x_{r}, y_{r}, P_{1}, P_{2}\right)-q P_{S R D}\left(P_{1}, P_{2}\right) \\
\text { subject to } & \mathrm{C} 1, \mathrm{C} 2, \mathrm{C} 3 .
\end{array}
$$

Since the problem in (19) is a strictly quasi-concave optimization problem, we can solve it by using the Lagrange multiplier method with Karush-Kuhn-Tucker (KKT) conditions [11]. The Lagrangian of (19) can be given by

$$
\begin{aligned}
& \mathcal{L}\left(x_{r}, y_{r}, P_{1}, P_{2}, \mu_{1}, \mu_{2}, \mu_{3}\right) \\
= & C_{S R D}\left(x_{r}, y_{r}, P_{1}, P_{2}\right)-q P_{S R D}\left(P_{1}, P_{2}\right)-\mu_{1}\left(P_{1}+P_{2}-P_{s}\right) \\
& -\mu_{2}\left(\left(x_{r}+\frac{d_{S D} \sin \theta}{2}\right)^{2}+y_{r}^{2}-\left(\frac{d_{S D} \sin \theta}{2}\right)^{2}\right) \\
& -\mu_{3}\left(x_{r}-\delta\right),
\end{aligned}
$$

TABLE II

The Proposed Iterative Algorithm

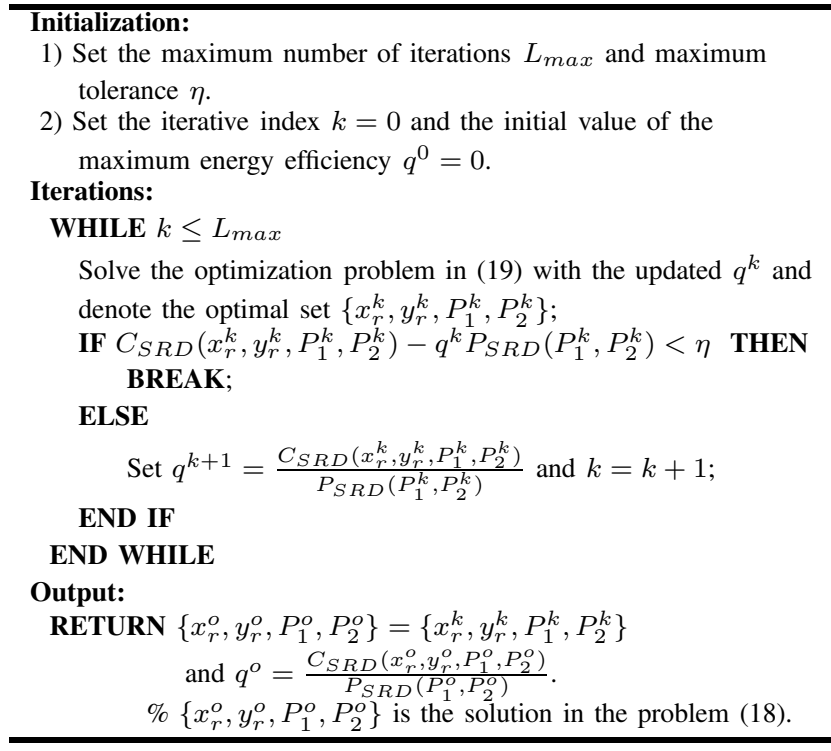

where $\mu_{1}, \mu_{2}, \mu_{3} \geq 0$ are the Lagrange multipliers connected to C1-C3. We define $\left\{\tilde{x}_{r}, \tilde{y}_{r}, \tilde{P}_{1}, \tilde{P}_{2}\right\}$ as the solution of the problem (19). Considering the KKT conditions and taking the stationarity condition of each variable, we can prove that $\mu_{2}=$ $\mu_{3}=0$ and the solution $\left\{\tilde{x}_{r}, \tilde{y}_{r}, \tilde{P}_{1}, \tilde{P}_{2}\right\}$ meets (21)-(24) with a given $q$, e.g.,

$$
\tilde{y}_{r}=0
$$

$$
\frac{\tilde{x}_{r} n_{0} M_{0} \tilde{P}_{2} \xi_{R D}}{n_{1} M_{1} \tilde{P}_{1} \xi_{S R}} A_{1}\left(\tilde{x}_{r}, 0\right)+\left(\tilde{x}_{r}+d_{S D} \sin \theta\right) A_{2}\left(\tilde{x}_{r}, 0\right)=0,
$$

$$
\begin{aligned}
A_{3} & +\frac{\xi_{S R} \xi_{R D} A_{4}\left(\tilde{x}_{r}, 0\right) \tilde{P}_{2}^{2}}{\left(\tilde{P}_{2} A_{4}\left(\tilde{x}_{r}, 0\right)+\tilde{P}_{1} A_{5}\left(\tilde{x}_{r}, 0\right)\right)^{2}} \\
= & \frac{\xi_{S R} \xi_{R D} A_{5}\left(\tilde{x}_{r}, 0\right) \tilde{P}_{1}^{2}}{\left(\tilde{P}_{2} A_{4}\left(\tilde{x}_{r}, 0\right)+\tilde{P}_{1} A_{5}\left(\tilde{x}_{r}, 0\right)\right)^{2}}
\end{aligned}
$$

and

$$
\frac{\frac{1}{2 \sigma_{n}^{2}} \frac{\xi_{S R} \xi_{R D} A_{5}\left(\tilde{x}_{r}, 0\right) \tilde{P}_{1}^{2}}{\left(\tilde{P}_{2} A_{4}\left(\tilde{x}_{r}, 0\right)+\tilde{P}_{1} A_{5}\left(\tilde{x}_{r}, 0\right)\right)^{2}}}{1+\frac{N_{f}}{\sigma_{n}^{2}} \tilde{P}_{1} A_{3}+\frac{N_{f}}{\sigma_{n}^{2}} \frac{\xi_{S R} \xi_{R D} \tilde{P}_{1} \tilde{P}_{2}}{\tilde{P}_{2} A_{4}\left(\tilde{x}_{r}, 0\right)+\tilde{P}_{1} A_{5}\left(\tilde{x}_{r}, 0\right)}}=\ln (2)\left(\frac{\varepsilon q}{2}+\mu_{1}\right),
$$

where $A_{1}\left(x_{r}, y_{r}\right)=\left(x_{r}^{2}+y_{r}^{2}\right)^{\frac{n_{0}-2}{2}}$ and $A_{2}\left(x_{r}, y_{r}\right)=$ $\left(\left(x_{r}+d_{S D} \sin \theta\right)^{2}+y_{r}^{2}+d_{r}^{2}\right)^{\frac{n_{1}-2}{2}} \cdot A_{3}=\frac{\xi_{S D}}{M_{1} d_{S D}^{n_{1}}}$ is constant. $A_{4}\left(x_{r}, y_{r}\right)=\xi_{R D} M_{0}\left(x_{r}^{2}+y_{r}^{2}\right)^{\frac{n_{0}}{2}}$ and $A_{5}\left(x_{r}, y_{r}\right)=$ $\xi_{S R} M_{1}\left(\left(x_{r}+d_{S D} \sin \theta\right)^{2}+y_{r}^{2}+d_{r}^{2}\right)^{\frac{n_{1}}{2}}$.

1) Discussion: With (21)-(24), we can see that the optimal relay location is always located on the $\mathrm{x}$-axis in the proposed model. Besides, it is revealed that the value of $x_{r}^{o}$ and $\alpha=$ $P_{1}^{o} / P_{2}^{o}$ are determined by the given $d_{S D}$ and $d_{r}$ and they are independent of $q$ and $P_{s}$. In (24), it is noted that whether the 
value of $\mu_{1}$ is equal to 0 or not depends on values of $P_{s}$ and $q$. Based on KKT conditions, $\tilde{P}_{1}+\tilde{P}_{2}<P_{s}$ when $\mu_{1}=0$ and $\tilde{P}_{1}+\tilde{P}_{2}=P_{s}$ when $\mu_{1}>0$. For the along-torso scenario, the related problem can be also formulated and the joint optimal RL and PA can be achieved with the same iterative algorithm and a similar derivation.

2) Implementation: A typical application for the aroundtorso scenario in WBANs is the post-neck surgery tracking for patients, where a sensor node is placed on the neck of a patient to measure the angular motion of the neck and send the updated status of recovery to the coordinator in the front pocket. The proposed joint optimal RL and PA scheme aims to provide an insight into the design of healthcare applications with respect to the proper placement of the wearable relay node along with the optimal transmit power level in WBANs. In some cases, with high sensor node density in a WBAN, it is inappropriate to setup additional relay nodes for the sensor nodes and available sensor node can be selected as a relaying node to cooperate in forwarding the data from one node towards the coordinator. Thus, this work can be also considered as a source of inspiration for a WBAN relay selection protocol. It is a guideline for the coordinator to assign an available node with proper location and transmit power to assist a given transmit node when the network topology is known at the coordinator.

\section{Simulation}

To evaluate the energy efficiency of the proposed scheme in UWB based WBANs, numerical results are conducted in this Section. In simulations, $T_{w}$ and $T_{f}$ are chosen to be $2 \mathrm{~ns}$ and $150 \mathrm{~ns}$, respectively. $N_{f}$ is set to be 4 . The noise power spectral density (PSD) is $-174 \mathrm{dBm} / \mathrm{Hz}$ and the system bandwidth is $500 \mathrm{MHz}$. Since the average FCC PSD emission limit for UWB signals is $-41.3 \mathrm{dBm} / \mathrm{MHz}$, the maximum average transmit power $P_{\text {ave }}$ is $-14.3 \mathrm{dBm}$. With the duty cycle $T_{w} / T_{f}, P_{\max }=P_{\text {ave }} * T_{f} / T_{w}=4 \mathrm{dBm}$. In addition, $\varepsilon$ is set to be $2 . P_{c t}$ and $P_{c r}$ are set to be $100 \mu \mathrm{W}$ and $150 \mu \mathrm{W}$, respectively. According to the scale of the human body, $d_{S D}$ is very limited and meets that $0.5 \mathrm{~m} \leq d_{S D} \leq 0.8 \mathrm{~m}$. At $R$ and $D$, we assume that all of the dispersive energies can be captured without considering the ISI.

TABLE III

$\left\{x_{r}^{o}, y_{r}^{o}\right\}$ AND $\alpha=P_{1}^{o} / P_{2}^{o}$ FOR BOTH SCENARIOS

\begin{tabular}{|c|c|c|c|c|}
\hline \multicolumn{5}{|c|}{ Along-torso scenario $\left(d_{r}=0\right)$} \\
\hline$d_{S D}$ & $0.5 \mathrm{~m}$ & $0.6 \mathrm{~m}$ & $0.7 \mathrm{~m}$ & $0.8 \mathrm{~m}$ \\
\hline$x_{r}^{O}$ & -0.30 & -0.36 & -0.42 & -0.48 \\
\hline$y_{r}^{O}$ & 0 & 0 & 0 & 0 \\
\hline$\alpha$ & 2.37 & 2.37 & 2.37 & 2.37 \\
\hline \hline \multicolumn{5}{|c|}{ Around torso-scenario with $d_{r}=0.2 \mathrm{~m}$} \\
\hline$d_{S D}$ & $0.5 \mathrm{~m}$ & $0.6 \mathrm{~m}$ & $0.7 \mathrm{~m}$ & $0.8 \mathrm{~m}$ \\
\hline$x_{r}^{O}$ & -0.42 & -0.52 & -0.62 & -0.72 \\
\hline$y_{r}^{O}$ & 0 & 0 & 0 & 0 \\
\hline$\alpha$ & 0.74 & 1.02 & 1.32 & 1.64 \\
\hline
\end{tabular}

Table III presents the optimal relay location $\left\{x_{r}^{o}, y_{r}^{o}\right\}$ and power ratio $\alpha=\frac{P_{1}^{o}}{P_{2}^{o}}$ of the proposed scheme with various values of $d_{S D}$ for both scenarios. It is shown that the optimal relay is always located on the negative $\mathrm{x}$-axis for both scenarios and it is very close to the point $P$ in the aroundtorso scenario. For the along-torso scenario, we can see that the power ratio $\alpha$ is invariant with different $d_{S D}$. This is due to the fact that, since in the along-torso scenario, all signals are transmitted over the along torso channels, the optimal relay location relative to $S$ and $D$ is unchanged when $d_{S D}$ varies. Thus $\alpha$ does not vary. Different from the along-torso scenario, $\alpha$ varies with $d_{S D}$ in the around-torso scenario. This can be explained by the fact that signals are transmitted over the along-torso channel only for the $S$ - $R$ link and over the around-torso channels for the other two links. The change of $d_{S D}$ has an impact on the optimal relay location relative to $S$ and $D$ and therefore $\alpha$.

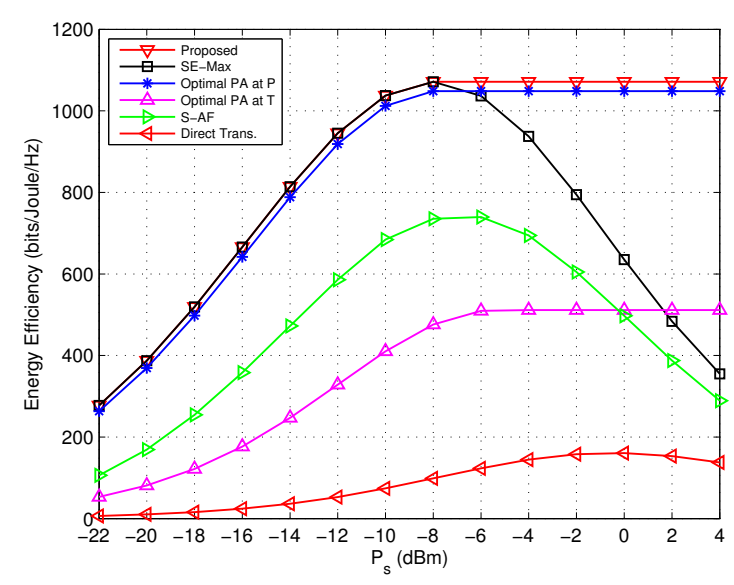

Fig. 2. Energy efficiency versus $P_{s}$ in the around-torso scenario with $d_{S D}=$ $0.6 \mathrm{~m}$ and $d_{r}=0.2 \mathrm{~m}$

Fig. 2 depicts the average energy efficiency versus $P_{s}$ in the around-torso scenario. Five transmission schemes are illustrated in this figure to compare with the proposed joint optimal PA and RL scheme, which are the SE-maximizing (SE-Max) scheme, the optimal PA scheme at point $P$, the optimal PA scheme at point $T$, selective AF (S-AF) scheme, and direct transmission, respectively. In the SE-Max scheme, the SE in (17) with constraints $\mathrm{C} 1-\mathrm{C} 3$ is maximized. In the optimal PA schemes at points $P$ and $T$, the optimal $P_{1}$ and $P_{2}$ are exploited to maximize the energy efficiency when $R$ is fixed at points $P$ and $T$, respectively. In the $\mathrm{S}-\mathrm{AF}$ scheme, we assume that 5 relays are randomly located in the circle defined in $\mathrm{C} 1$ and the relay that can achieve the maximum energy efficiency is selected. As shown in this figure, the proposed scheme outperforms all the other schemes. The optimal energy efficiency and SE can be achieved simultaneously by the proposed scheme when $P_{s}$ is in the low-to-moderate regimes. As $P_{s}$ increases in the moderate-to-high regimes, the energy efficiency of the SE-Max scheme decreases rapidly, which can be explained by the fact that the SE-Max scheme always uses the maximum power for capacity maximization which is harmful for the energy efficiency. Compared to direct transmission, 
we can see that the proposed scheme can provide a remarkable performance improvement and up to 30 times improvement can be achieved when the battery of the sensor node is very limited (e.g., $P_{s} \leq-20 \mathrm{dBm}$ ). This evidence indicates that the lifetime of WBANs can be prolonged considerably by using the proposed scheme. Furthermore, it is noticed that a large performance gap exists between the optimal PA schemes at points $P$ and $T$ and the energy efficiency of the optimal PA scheme at point $P$ is very close to the optimum, which matches our analysis well in Table III. Based on these observations, we can see that the impact of the relay location cannot be ignored in cooperative transmission and placing the relay close to $P$ is a good option for achieving high energy efficiency.

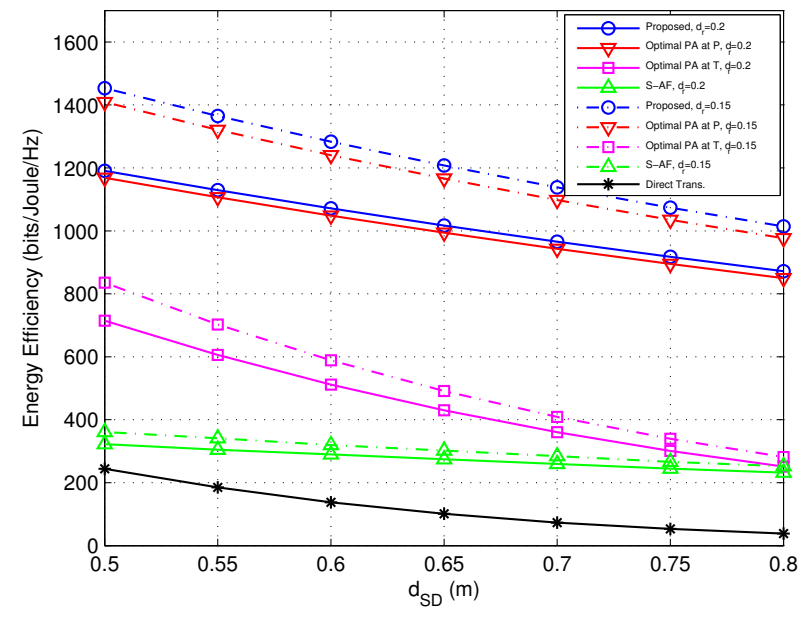

Fig. 3. Energy efficiency versus $d_{S D}$ in the around-torso scenario with $P_{s}=$ $4 \mathrm{dBm}$.

Fig. 3 illustrates the average energy efficiency versus $d_{S D}$ with fixed $P_{s}=4 \mathrm{dBm}$ in the around-torso scenario. Two cases with $d_{r}=0.15 \mathrm{~m}$ and $d_{r}=0.2 \mathrm{~m}$ are considered, respectively. We notice that direct transmission is sensitive to $d_{S D}$ and it has a poor performance when $d_{S D}$ is large. That is to say, without the line of sight (LoS) between $S$ and $D$, the significant propagation loss would affect the performance of direct transmission adversely. By contrast, the proposed scheme exhibits a weak dependence upon $d_{S D}$, which indicates that the proposed scheme is helpful for the robustness against the around-torso based path loss and can be an effective way for the extension of transmission range.

In Fig. 4, the average energy efficiency versus $P_{s}$ with different $d_{S D}$ in the along-torso scenario $\left(d_{r}=0\right)$ is depicted. Different from results in Fig. 2, direct transmission is more energy efficient than the proposed scheme when $P_{s}$ is in the low-to-moderate regimes. This is because when a LoS between $S$ and $D$ is present, the path loss exponent is small, and thus the circuit power dissipation dominates the performance of the energy efficiency. Since cooperative transmission costs more circuit power, it would never take advantage in this case.

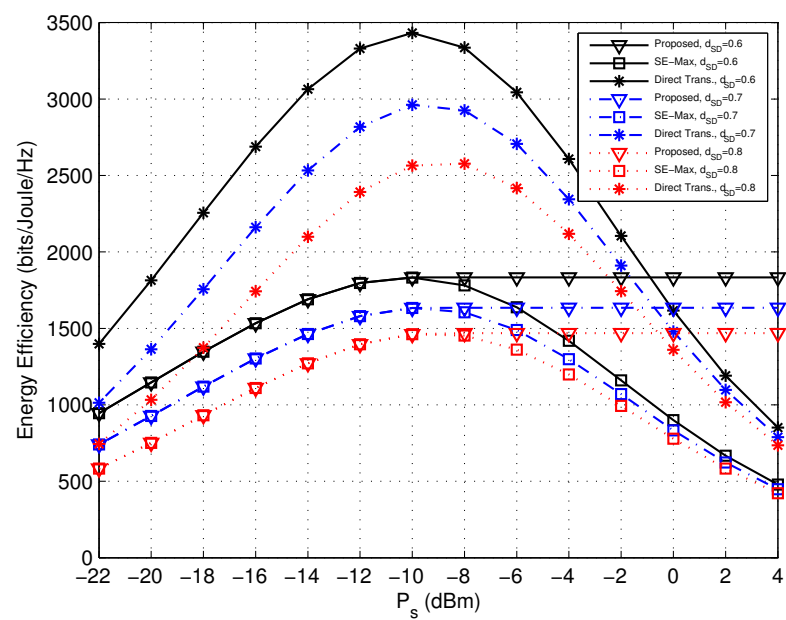

Fig. 4. Energy efficiency versus $P_{s}$ in the along-torso scenario.

\section{CONCLUSIONS}

In this paper, we investigate the energy efficiency of singlerelay cooperative transmission in UWB based WBANs. The joint optimal relay location and power allocation are derived and analyzed for the energy efficiency maximization. Numerical results show that the proposed scheme is superior to other schemes and it is an effective way to prolong the lifetime of WBANs and extend the transmission range in WBANs, particularly for the around-torso scenario. This work can be easily extended to wireless implant-body area networks and multiple-relay case in WBANs will be considered in future.

\section{REFERENCES}

[1] IEEE Standard for local and metropolitan area networks-Part 15.6: Body Area Networks, 2012.

[2] Z. Zhou, S. Zhou, J-H. Cui, and S. Cui, "Energy-efficient cooperative communication based on power control and selective single-relay in wireless sensor networks," IEEE Transactions on Wireless Communications, vol. 7, no. 8, Aug. 2008, pp. 3066-3078.

[3] A. K. Sadek, W. Yu, and K. J. R. Liu, "On the energy efficiency of cooperative communications in wireless sensor networks," ACM Transactions on Sensor Networks, vol. 6, no. 1, Dec. 2009, pp. 1-21.

[4] G. Li, Z. Xu, C. Xiong, C. Yang, S. Zhang, Y. Chen, and S. Xu, "Energyefficient wireless communications: tutorial, survey, and open issues," IEEE Wireless Communications, vol. 18, no. 6, 2011, pp. 28-35.

[5] A. Kailas, "Power allocation strategies to minimize energy consumption in wireless body area networks," IEEE EMBS, Aug. 2011, pp. 2204-2207

[6] X. G. Huang, H. G. Shan, and X. M. Shen, "On energy efficiency of cooperative communications in wireless body area networks," IEEE WCNC, 2011, pp. 1097-1101.

[7] G. Ntouni, A. Lioumpas, and K. Nikita, "Reliable and energy efficient communications for wireless biomedical implant systems," IEEE Journal of Biomedical and Health Informatics, 2014, pp. 1-8.

[8] J. Elias, "Optimal design of energy-efficient and cost-effective wireless body area networks," Ad Hoc Networks, vol. 13, 2014, pp. 560-574.

[9] K. Y. Yazdandoost and K. Sayrafian-Pour, "Channel model for body area network (ban)," IEEE P802.15 Working Group for Wireless Personal Area Networks (WPANs), Tech. Rep. Document IEEE802.15-08-078005-0006, February 2009.

[10] W. Dinkelbach, "On nonlinear fractional programming," Management Science, vol. 13, no. 7, March 1967, pp. 492-498.

[11] S. Boyd and L. Vandenberghe, Convex Optimization. Cambridge University Press, 2004. 